Bond University

Research Repository

\title{
Deliberate clinical inertia: Using meta-cognition to improve decision-making
}

Keijzers, Gerben; Fatovich, Daniel M.; Egerton-Warburton, Diana; Cullen, Louise; Scott, lan A.; Glasziou, Paul; Croskerry, Pat

Published in:

EMA - Emergency Medicine Australasia

DOI:

$10.1111 / 1742-6723.13126$

Licence:

Other

Link to output in Bond University research repository.

Recommended citation(APA):

Keijzers, G., Fatovich, D. M., Egerton-Warburton, D., Cullen, L., Scott, I. A., Glasziou, P., \& Croskerry, P. (2018). Deliberate clinical inertia: Using meta-cognition to improve decision-making. EMA - Emergency Medicine Australasia, 30(4), 585-590. https://doi.org/10.1111/1742-6723.13126

\section{General rights}

Copyright and moral rights for the publications made accessible in the public portal are retained by the authors and/or other copyright owners and it is a condition of accessing publications that users recognise and abide by the legal requirements associated with these rights.

For more information, or if you believe that this document breaches copyright, please contact the Bond University research repository coordinator. 
DELIBERATE CLINICAL INERTIA:

Using meta-cognition to improve decision-making

Gerben Keijzers MSc (Biomed Health Sci) MBBS FACEM PhD, Department of Emergency Medicine, Gold Coast University Hospital, Gold Coast, QLD, Australia; School of Medicine, Bond University, Gold Coast, QLD, Australia; School of Medicine, Griffith University, Gold Coast, QLD, Australia

Daniel M Fatovich MBBS FACEM PhD, Professor of Emergency Medicine, Royal Perth Hospital, University of Western Australia; Head, Centre for Clinical Research in Emergency Medicine, Harry Perkins Institute of Medical Research

Diana Egerton-Warburton MBBS FACEM MClinEpi MPH, School of Clinical Science at Monash Health, Monash University Faculty of Medicine Nursing and Health Sciences, Melbourne Victoria, Australia.

Louise Cullen MBBS (Hon) FACEM PhD, Emergency and Trauma Centre, Royal Brisbane and Women's Hospital, Queensland University of Technology, University of Queensland, Brisbane, QLD, Australia

Ian A. Scott MBBS FRACP MHA, Director of Internal Medicine and Clinical Epidemiology, Princess Alexandra Hospital; Associate Professor of Medicine, University of Queensland, Brisbane, Australia

Paul Glasziou MBBS, FRACGP, PhD, Director Centre for Research in Evidence-Based Practice, Bond University, Queensland, Australia

Pat Croskerry MD, PhD, FRCP(Edin). Professor of Emergency Medicine and Director of the Critical Thinking Program, Dalhousie University, Halifax, Nova Scotia, Canada.

Abstract: 108 Word count: 2230 References: 29

Key words: clinical decision making, cultural change, human factors, overdiagnosis,

\section{Competing interests:}

GK and DEW are section editors for Emergency Medicine Australasia 
DELIBERATE CLINICAL INERTIA

Using meta-cognition to improve decision-making

\begin{abstract}
Deliberate clinical inertia is the art of doing nothing as a positive response. To be able to apply this concept, individual clinicians need to specifically focus on their clinical decision making. The skill of solving problems and making optimal clinical decisions requires more attention in medical training and should play a more prominent part of the medical curriculum. This paper provides suggestions on how this may be achieved. Strategies to mitigate common biases are outlined, with an emphasis on reversing a 'more is better' culture towards more temperate, critical thinking. To incorporate such an approach in medical curricula and in clinical practice, institutional endorsement and support is required.
\end{abstract}




\section{DELIBERATE CLINICAL INERTIA}

\section{Using meta-cognition to improve decision-making}

\section{Introduction}

This is the third and final instalment reflecting on our clinical practice and clinical decision making. In the first paper we coined the term 'deliberate clinical inertia' and provided suggestions on how to achieve a balance between under- and overdoing in clinical care. The second paper highlighted several clinical scenarios where deliberate clinical inertia could be implemented in our practice. We suggested approaches for common clinical scenarios that would improve patient outcomes and the clinician's experience, as well as reduce healthcare cost. Using a case study, this last paper will focus on strategies for enhancing clinical reasoning and decision-making skills that foster deliberate clinical inertia.

\section{A case of lower limb cellulitis}

A 44-year-old accountant presents in the late afternoon to the emergency department (ED) with leg cellulitis. She was working in the garden a few days previously and sustained a scratch to her lower leg. It became red and tender and she decided to go to the ED since she could not get into the GP late in the day. Despite a low-grade fever (temperature $37.7^{\circ} \mathrm{C}$ ), she is systemically well, with no rigors, nausea or vomiting. She is otherwise healthy, with no history of diabetes or immunocompromise. It is busy at triage and since it will be a few hours to be seen, a peripheral intravenous cannula (PIVC) is inserted ("just in case she needs intravenous antibiotics, plus she needs blood tests anyway, doesn't she?") and blood is sent for testing including a CRP. When the patient is assessed by the treating doctor, the working diagnosis of cellulitis is made. Since the patient has a PIVC in place, the decision is made to give 2 grams of intravenous flucloxacillin in the ED short stay ward ("She already has a cannula, right? - it is quite red and may be early sepsis, can't hurt - can it?'). The treating doctor hands over the care to the short stay team, and since the patient turnover in short stay is high, the patient is not reviewed until after 10PM, where the decision is made that she may as well stay overnight for a few more intravenous 
doses of antimicrobials. ("It is a probably a good idea, since the CRP was elevated at 43"). The next morning, the patient is still well without systemic features and she is discharged just before lunch with a prescription for oral flucloxacillin and a medical certificate for work, 18 hours after presenting.

\section{Cognitive bias and metacognition}

The case described is not unusual. The human mind has the tendency to deal with problems in simple ways. It aims to minimise cognitive effort and avoid use of unnecessary mental bandwidth: a phenomenon that has been called 'the cognitive miser function' by Fiske et al. ${ }^{1}$ In an ideal world this patient would not have had a PIVC inserted, not undergone certain blood tests, not received intravenous antibiotics, nor be required to have a prolonged overnight stay in ED and miss the next day at work. Individual clinicians need to be more aware of their decision making, and the skill of solving problems and making optimal clinical decisions requires more attention in medical training.

At Dalhousie University Medical School (Halifax, Canada) a program in critical thinking was established in 2012 and from the start of medical training, students are exposed to the dominant model for decision making: Dual Process Theory, ${ }^{2}$ which consists of fast (Type 1 - intuitive decisions) and slow (Type 2 - analytical) processing. Although implemented as a 'critical thinking' program, the main impetus was to raise the profile of clinical reasoning and decision making in undergraduate and postgraduate medical training. The dominant theme of the program is rationality and how brain 'mindware'3 is responsible for both the content and processing of information. Students are educated about factors that both inhibit rationality (lack of insight into the decision making process, cognitive and affective bias, cognitive miserliness, logical fallacies, failures to think scientifically and critically) as well as promote it (medical knowledge, critical thinking, metacognition, mindfulness, reflection, and bias mitigation strategies). Other important characteristics of the overall clinical assessment and management process are included such as ordering 
and interpretation of tests, communication strategies, and shared decision making with the patient.

\section{Deliberate clinical inertia as part of the curriculum}

Thoughtful awareness and thinking about our thinking (meta-cognition) and actions are essential in changing clinician behaviour in a meaningful and sustainable way. It requires ongoing engagement and dedicated effort of all relevant stakeholders, since so many behaviours are routine and ingrained.

The current Australian Curriculum Framework for Junior Doctors ${ }^{4,5}$ has a heading 'decision making', which consists of three areas:

- 'Explain the indications, contraindications and risks for common procedures'

- 'Select appropriate procedures with involvement of senior clinicians and the patient'

- 'Consider personal limitations and ensures appropriate supervision'

Unfortunately, these statements do not clearly define the skills necessary for making wise decisions that take account of limited information and knowledge, and commonly encountered cognitive heuristics and biases. They also neglect the importance of emotions, the environmental pressures under which decisions must be made, and the need to communicate potential benefits and harms of future actions as part of shared decision making.

The study and practice of clinical decision making should be a prominent part of the medical curriculum (which is possible as evidenced at Dalhousie), and specialty training programs can tailor the content to meet the needs of their particular specialty. Instilling a meta-cognitive perspective of reflection and selfregulation into everyday practice will encourage every clinical decision to be actively thought through and lessen the risk of cognitive error arising from biased, intuitive ("Type 1") decision making. This need not entail an exhaustive analytical approach to every decision a clinician makes, as Type 1 decision making, reliant on heuristics (mental short-cuts or rules of thumb), will usually 
suffice. However, well-calibrated decision makers should always reserve the option to examine their Type 1 decisions deliberately and analytically (using "Type 2" processing) when the need is apparent. How might these themes be accommodated within medical education?

Firstly, our curricula (formal, informal, and hidden) and learner assessment methods need to be modified to emphasise reasoning. Ideally this includes the content of clinical problems and the context in which they occur, the existence of multiple diagnostic possibilities and treatment options, and the need to consider contextual factors and patient perspectives and values in arriving at final decisions. ${ }^{6,7}$

Secondly, education must emphasise that uncertainty is the natural order. Doctors frequently make decisions on the basis of imperfect and incomplete data, which results in diagnostic and therapeutic uncertainty. Indeed, it is instructive to note that most doctors make patient-centered decisions every day without high-quality evidence, and that these decisions can be adjusted in response to developing insights or new information. Furthermore, foundational papers in evidence-based medicine (EBM) make it explicitly clear that EBM never intended to exclude information derived from experience and intuition. ${ }^{8}$ Diagnosis is also a dynamic process, and delay in diagnosis is part of the natural course of many conditions. This natural diagnostic process leaves clinicians often with multiple reasonable and defensible investigation and management decisions of which the outcomes cannot always be predicted with confidence. The interplay of patient preferences, societal values, logistical constraints, and resource availability adds to the complexity. Developing and maintaining a tolerance for uncertainty, curiosity about the unknown, and skills in managing uncertainty are keys to becoming (and remaining) a resilient, effective clinician. Actively promoting discussions that embrace the grey zones of medicine (areas lacking definitive evidence to guide clinical actions) and developing students' (and clinicians') ability to explicitly acknowledge and be comfortable with uncertainty must be an essential part of contemporary medical curricula. ${ }^{6}$ 
Thirdly, there must be open exploration of how reasoning may falter. One method for increasing awareness and understanding of one's own thought process (meta-cognition) is to engage in cognitive huddles (or "time out") ${ }^{9}$ whereby students and experienced clinicians discuss perplexing or difficult cases and disclose actual or potential missteps in decision making related to both cognitive (internal) and non-cognitive (external) factors. Recounting and thinking through a reasoning error with students and doctors (of any seniority) enhances everyone's learning and ability to explore their own thought processes more deeply within a psychologically safe environment. Such an exercise also empowers clinicians to ask their seniors or peers to explain the reasoning behind their decisions and, in appropriate circumstances, encourage deliberate clinical inertia. ${ }^{10,11}$

Fourthly, the commonly encountered biases in decision-making must be exposed and strategies for countering them emphasised. Our case study exemplifies several of them. A list of common biases and descriptors are summarised in Table 1, with more described elsewhere..$^{9,12,13}$ Misdiagnosis or delayed diagnosis of serious conditions loom large as matters of concern for both patient and clinician. Several bias mitigating strategies (see Table 2), if explicitly taught and regularly practised, may render clinicians less vulnerable to erroneous decisions resulting from biased reasoning. These strategies can be focused on both diagnostic and treatment processes.

In the case study, the possibility of cellulitis becoming severe sepsis ("Don't' chase zebras" - Table 2 , row 2$)^{12}$ if the patient was not treated aggressively with intravenous antibiotics and monitored in hospital were key drivers of clinical actions. Omission regret is a strong motivator for clinicians to do things rather than stand still and think more deeply. ${ }^{14}$ Clinicians worry more about seeing a patient suffer a bad but unlikely outcome they might have been able to prevent by doing something ("tolerating uncertainty") 6 . They worry less about the negative consequences of giving an unnecessary treatment to many more people who stand to gain no benefit. What may help in such situations is for clinicians to gain confidence in thinking more probabilistically (by balancing the likelihood of the diagnosis they fear most versus estimating 
and noting the likelihood of the diagnosis most favoured given all the clinical features in the medical record- "probabilistic notation') ${ }^{15}$ and acting expectantly by treating the condition most favoured and reassess as circumstances evolve ("slow diagnosis") ${ }^{16}$. In the case study, uncomplicated mild cellulitis in a young immunocompetent patient is the most likely diagnosis based on clinical presentation and can be treated expectantly with oral antibiotics ("stepped care") 9,17 and advice to the patient to return if the condition worsens, with explicit time-specific and action-specific instructions ("safety netting"). 9,18,19

The other features of the case are the diagnostic momentum, ${ }^{12}$ framing effect ${ }^{12}$ and groupthink which fed into the ensuing cascade of overtreatment administered by a sequence of different clinicians. The hypothesis of sepsis was not challenged, the patient was framed as someone who needed inpatient care by virtue of having a PIVC inserted with intravenous antibiotics and blood tests, and groupthink meant that no-one prioritised the patient as being eligible for early reassessment and expedited discharge, and no-one altered their work flow accordingly.

Finally, we must acknowledge that clinicians, like all humans, are influenced by peer opinions and social norms. The ethicist John Banja describes the normalisation of deviance' as the large-scale acceptance and tacit endorsement of violations of recognised standards of practice as a factor contributing to preventable patient harm. ${ }^{20}$ This 'normalisation of deviance' could contribute to the over-testing and over-treatment that has been described over the last 20 years. For example, in the last decade, the proportion of patients with suspected renal colic who undergo CT scans of the kidneys, ureters and bladder has progressively increased from $25 \%$ to $75 \%{ }^{21}$ If this increase had occurred rapidly, clinicians would interpret it as an unwarranted variation from standard practice, but as it has occurred gradually it has become accepted as not only normal but as standard practice. Greater recognition of 
indication creep for both tests and treatments may provide an opportunity to recalibrate what the average clinician regards as normal.

Reversing this 'more is better' culture towards more temperate, critical thinking will need professional endorsement, especially by influential senior opinion leaders, good role models, and institutional support. Changing behaviour of groups of clinicians and whole organisations is challenging to enact and to measure, especially with regards to quantity and quality of clinical decision making. The professionally-led Choosing Wisely initiative, ${ }^{22}$ which was launched to improve stewardship of resource use by pinpointing areas of low-value care, has made limited inroads in most of the areas that have been investigated in the United States to date..$^{23}$ Achieving cultural change is further complicated if new initiatives impact on other performance indicators, such as compliance with established guidelines. This especially relevant for guidelines which pursue a more aggressive approach to care, including recommendations for multiple interventions, and fail to detail clinical circumstances where recommendations may not be applicable. Patients require nuanced decisions and there must be room for practice variation, based upon special patient characteristics, and shared decision making. Institutions such as hospitals, governments, and universities need to support judicious, patient-centred deviation from recommendations in guidelines that are based on low level evidence. To engage and empower clinicians, guideline panels might consider reasons for such deviations in guideline development or updates.

In conclusion, in busy everyday practice, our heuristics and habits save cognitive effort compared to more analytical thinking. However, clinicians need to retain situational awareness and actively consider alternative ideas that may go against the 'flow'. Developing skills in dealing concurrently with ambiguity, competing diagnostic or management options, harm-benefit trade-offs, system of care exigencies and social pressures is paramount in developing deliberate clinical inertia. This will require explicit training in both undergraduate and 
postgraduate curricula, and change leadership from senior clinicians, organisational leaders and guideline developers. 


\section{References}

1. Fiske et al. Social cognition (2nd ed.). New York: Mcgraw-Hill. ISBN 0070211914. Fiske ST, Taylor SE

2. Croskerry P. A model for clinical decision making in medicine. Medical Science Educator 2018 https://doi.org/10.1007/s40670-017-0499-9

3. Perkins D. Outsmarting IQ: The emerging science of learnable intelligence. New York: the Free Press, 1995

4. Australian Medical Council, part of Australian Curriculum Framework for Junior Doctors (ACF). Available at URL:

http://www.cpmec.org.au/acf-2010/index.cfm Last accessed 30 April 2018

5. Australian Medical Council, part of Australian Curriculum Framework for Junior Doctors (ACF). Available at URL:

http://www.cpmec.org.au/ACF-2010/clinicalmanagement.cfm Last accessed 30 April 2018

6. Simpkin AL, Schwartzstein RM. Tolerating uncertainty, the next medical revolution? N Engl J Med 2016; 375:1713-1715

7. Brand G, Wise S, Siddiqui ZS, Celenza A, Fatovich DM. Capturing the 'art' of emergency medicine: Does film foster reflection in medical students? Emerg Med Australas. 2017;29:433-7.

8. Braithwaite RS. A piece of my mind. EBM's six dangerous words. JAMA 2013;310:2149-50.

9. Scott IA, Soon J, Elshaug AG, Lindner R. Countering cognitive biases in minimising low value care. Med J Aust. 2017;206(9):407-411.

10. Xie Y. Medical student enquiries on the art of clinical inertia. Emerg Med Australas. 2018 Mar 30. doi: 10.1111/1742-6723.12973.

11. Keijzers G, Cullen L, Egerton-Warburton D, Fatovich DM. Re: Medical student enquiries on the art of clinical inertia. Emerg Med Australas. 2018 Apr 15. doi: 10.1111/1742-6723.12980.

12. Croskerry $P$. Achieving quality in clinical decision making: cognitive strategies and detection of bias. Acad Emerg Med. 2002 Nov;9(11):1184-204. 
13. Scott IA. Errors in clinical reasoning: causes and remedial strategies. BMJ 2009; 339: 22-25.

14. Ayanian JZ, Berwick DM. Do physicians have a bias toward action? A classic study revisited. Med Decis Making 1991; 11: 154-158.

15. Morgenstern J. Embracing the uncertainty of diagnosis. Emergency Medicine News. March 2015. Page 25

16. McCormack JP, Allan GM, Virani AS. Is bigger better? An argument for very low starting doses. CMAJ 201;1 183 (1) 65-69; DOI: https://doi.org/10.1503/cmaj.091481

17. Richards DA, Bower P, Pagel C, Weaver A, Utley M, Cape J et al. Delivering stepped care: an analysis of implementation in routine practice. Implement Sci. 2012 Jan 16;7:3. doi: 10.1186/1748-5908-7-3.

18. Almond S, Mant D, Thompson M. Diagnostic safety-netting. Br J Gen Pract. 2009 Nov;59(568):872-4; discussion 874.

19. Neighbour R. Safety netting: now doctors need it too. Br J Gen Pract. 2018;68(670):214-215.

20. Banja J. The normalization of deviance in healthcare delivery. Bus Horiz. 2010;53(2):139. doi:10.1016/j.bushor.2009.10.006

21. Blecher G, Meek R, Egerton-Warburton D, McCahy P. Introduction of a new imaging guideline for suspected renal colic in the ED reduces CT urography utilisation. Emerg Med J. 2017;34(11):749-54.

22. Cassel CK, Guest JA. Choosing wisely: helping physicians and patients make smart decisions about their care. JAMA 2012;307:18012

23. Rosenberg A, Agiro A, Gottlieb M, Barron J, Brady P, Liu Y, Li C, DeVries $A$. Early trends among seven recommendations from the Choosing Wisely Campaign. JAMA Intern Med. 2015 Dec;175(12):1913-20. doi: 10.1001/jamainternmed.2015.5441

24. Lenzer J, Hoffman JR, Furberg CD, loannidis JP. Ensuring the integrity of clinical practice guidelines: a tool for protecting patients. BMJ (Clinical research ed) 2013; 347: f5535. 
25. Gillis AM. Tincture of time: when to implant a prophylactic cardioverterdefibrillator following coronary revascularization? J Am Coll Cardiol. 2006; 47(9):1818-9

26. Probst MA, Kanzaria HK, Schoenfeld EM, Menchine MD, Breslin M, Walsh C, Melnick ER, Hess EP. Shared Decisionmaking in the Emergency Department: A Guiding Framework for Clinicians. Ann Emerg Med. 2017 Nov;70(5):688-695.

27. Kerr EA, Hofer TP. Deintensification of routine medical services: The next frontier for improving care quality. JAMA Intern Med 2016;176(7):978-80

28. Thaler RH. Nudge: Improving Decisions about Health, Wealth, and Happiness. Yale University Press. 2008. ISBN 9780143115267

29. Kassirer JP, Kopelman RI. Cognitive errors in diagnosis: instantiation, classification and consequences. Am J Med 1989;86:433-41. 
Table 1 - Common cognitive biases ${ }^{12}$

\begin{tabular}{|c|c|}
\hline Bias & Description \\
\hline $\begin{array}{l}\text { Anchoring and } \\
\text { adjustment }\end{array}$ & $\begin{array}{l}\text { Anchoring is the tendency to fixate on specific features of a } \\
\text { presentation too early in the diagnostic process, and to base the } \\
\text { likelihood of a particular event on information available at the } \\
\text { outset (i.e., this person may have early sepsis). This can be an } \\
\text { effective strategy. However, this initial impression exerts an overly } \\
\text { powerful effect in some people and they fail to adjust it sufficiently } \\
\text { in the light of later, potentially disconfirming information. }\end{array}$ \\
\hline Ascertainment & $\begin{array}{l}\text { Ascertainment bias occurs when the physician's thinking is pre- } \\
\text { shaped by expectations by what the physician specifically expects } \\
\text { to find. A physician is more likely to find evidence of congestive } \\
\text { heart failure in a patient who relates that he or she has recently } \\
\text { been noncompliant with his or her diuretic medication. Stereotyping } \\
\text { and gender biases are examples of ascertainment bias }\end{array}$ \\
\hline Availability & $\begin{array}{l}\text { Availability is the tendency for things to be judged more frequent if } \\
\text { they come readily to mind. Things that are common will be readily } \\
\text { recalled. The heuristic is driven by the assumption that the } \\
\text { evidence that is most available is the most relevant. Thus, if an } \\
\text { emergency physician previously saw a patient with cellulitis that led } \\
\text { to septic shock (and an ICU admission), there will be a greater } \\
\text { tendency to bring sepsis to mind when the next patient with } \\
\text { cellulitis presents. }\end{array}$ \\
\hline Confirmation & $\begin{array}{l}\text { When a hypothesis is developed on relatively weak or ambiguous } \\
\text { data, it may later interfere with superior and more plentiful data. } \\
\text { Such subsequent data might not be treated objectively and may be } \\
\text { ignored. Confirmation bias is reflected in a tendency to look for } \\
\text { confirming evidence to support the hypothesis, rather than look for } \\
\text { disconfirming evidence to refute it. ("Putting a square peg in a } \\
\text { round hole") }\end{array}$ \\
\hline $\begin{array}{l}\text { Diagnosis } \\
\text { momentum }\end{array}$ & $\begin{array}{l}\text { Diagnosis momentum refers to the tendency for a particular } \\
\text { diagnosis to become established without adequate evidence. } \\
\text { Typically, the process starts with an opinion, not necessarily a } \\
\text { medical one, of what the source of the patient's symptoms might } \\
\text { be. As this is passed from person to person, the diagnosis gathers } \\
\text { momentum to the point that it may appear almost certain by the } \\
\text { time the patient sees a physician. Attaching a diagnostic label is a } \\
\text { convenient short-hand way of communicating. It invariably means } \\
\text { that someone else's thinking has been inherited. Further dangers } \\
\text { imposed by this process are that it may result in further delays or } \\
\text { misdirection at ED triage. Physicians should always be wary when }\end{array}$ \\
\hline
\end{tabular}




\begin{tabular}{|c|c|}
\hline & $\begin{array}{l}\text { a patient begins the exchange by volunteering his or her own } \\
\text { diagnosis. }\end{array}$ \\
\hline Framing & $\begin{array}{l}\text { Framing occurs when equivalent descriptions of a scenario lead to } \\
\text { systematically different decisions depending on how they are } \\
\text { phrased. In our example people react differently to a patient with } \\
\text { 'possible sepsis' compared to 'a young healthy person with } \\
\text { cellulitis'. }\end{array}$ \\
\hline Overconfidence & $\begin{array}{l}\text { We usually think we know more than we do, often without having } \\
\text { gathered sufficient information, and generally place too much faith } \\
\text { in our own opinions. Those who are overconfident tend to spend } \\
\text { insufficient time accumulating evidence and synthesizing it before } \\
\text { action. They are more inclined to act on incomplete information and } \\
\text { hunches. When overconfident people believe that their involvement } \\
\text { might have a significant impact on outcomes (whether it actually } \\
\text { does or not), they tend to believe strongly that the outcome will be } \\
\text { positive. Thus, they disproportionately value their contribution. }\end{array}$ \\
\hline Premature closure & $\begin{array}{l}\text { Physicians typically generate several diagnoses early in their } \\
\text { encounter with a clinical problem. Premature closure occurs when } \\
\text { one of these diagnoses is accepted before it has been fully verified. } \\
\text { Attaching a diagnosis to a patient provides a convenient, short- } \\
\text { hand description. It may also reflect some laziness of thought and } \\
\text { a desire to achieve completion, especially when fatigued. }\end{array}$ \\
\hline Representativeness & $\begin{array}{l}\text { The patient's signs and symptoms are matched against the } \\
\text { physician's mental templates for their representativeness. Thus, we } \\
\text { often base our decision about whether or not something belongs to } \\
\text { a particular category by how well it matches the characteristics of } \\
\text { members of that category. The prototype is the most representative } \\
\text { member of the class. Traditionally medical education has taught } \\
\text { about prototype recognition. Thus, medical students are generally } \\
\text { more concerned with being able to list all the signs and symptoms } \\
\text { of unstable angina than list those "atypical" patients in whom the } \\
\text { diagnosis is occasionally missed. However, if we develop an } \\
\text { overreliance on the heuristic, we tend to misidentify atypical } \\
\text { variants of a category. }\end{array}$ \\
\hline Search Satisficing & $\begin{array}{l}\text { Search satisficing is the tendency to call off a search once } \\
\text { something is found. However, in the ED searching contingencies } \\
\text { are fundamentally different. There is often more than one thing to } \\
\text { be found, we are not always sure what it looks like, we do not } \\
\text { always know where to look, and we often do not find anything. In } \\
\text { many cases, satisfying oneself that the search is over once } \\
\text { something has been found will be erroneous }\left(2^{\text {nd }} \text { fracture, co- }\right. \\
\text { ingestion, } 2^{\text {nd }} \text { diagnosis). }\end{array}$ \\
\hline
\end{tabular}


Table 2.

Cognitive bias mitigation strategies for clinical decision-making in the following domains: i) Diagnostic and ii) Treatment/ Management at both individual and organisational level

\begin{tabular}{|l|l|}
\hline Strategy & Description \\
\hline DIAGNOSTIC & \\
\hline $\begin{array}{l}\text { Tolerating } \\
\text { uncertainty }\end{array}$ & $\begin{array}{l}\text { Understand situations where chasing complete or near certainty with } \\
\text { further investigations or trials of treatment is unnecessary and unlikely } \\
\text { to change management and may even distract from the main aim. } \\
\text { Seeking certainty may cause one to chase up blind alleys. }\end{array}$ \\
\hline $\begin{array}{l}\text { Don't chase } \\
\text { zebras }\end{array}$ & $\begin{array}{l}\text { Ask "what is the most likely diagnosis" and "what one diagnosis } \\
\text { shouldn't I miss?", with these in mind, use a parsimonious approach to } \\
\text { test ordering. This places a focus on estimating pre-test probability, } \\
\text { thinking in a Bayesian manner and applying the aphorism of Occam's } \\
\text { razor. }{ }^{12}\end{array}$ \\
\hline $\begin{array}{l}\text { Probabilistic } \\
\text { notation }\end{array}$ & $\begin{array}{l}\text { When documenting a diagnosis, clarify the level of confidence in this } \\
\text { diagnosis based on your assessment of its probability (likely/definite } \\
\text { diagnosis }>75 \%, \text { working diagnosis 50-75\%, uncertain but no other } \\
\text { diagnosis 10-50\%, unlikely <10\% but needs ruling out because of } \\
\text { critical impact) }\end{array}$ \\
\hline Time out & $\begin{array}{l}\text { Defer making a decision in non-urgent situations and take time to re- } \\
\text { think and research the problem when removed from workday pressures. } \\
\text { This technique is relevant to both awareness of knowledge deficits and } \\
\text { awareness of cognitive processing limitations, including fatigue. }\end{array}$ \\
\hline SREATMENT /
\end{tabular}




\begin{tabular}{|l|l|}
\hline $\begin{array}{l}\text { Shared } \\
\text { decision } \\
\text { making }\end{array}$ & $\begin{array}{l}\text { Patients and their clinicians reach agreement about goals of care. } \\
\text { Shared decision making is important in many scenarios where there is i) } \\
\text { clinical uncertainty or equipoise and ii) patient ability to make decisions. } \\
\text { An overarching goal is to avoid future (inappropriate) care, especially } \\
\text { invasive care.26 }\end{array}$ \\
\hline Stepped care & $\begin{array}{l}\text { Many initial assessments (outlier abnormalities of clinical or } \\
\text { physiological signs) will often return to normal, either due to the } \\
\text { intervention or because of the natural course of the condition. An } \\
\text { example of stepped care }{ }^{917} \text { is the initial prescription of analgesia for } \\
\text { uncomplicated childhood otitis media according to symptoms, with } \\
\text { delayed prescribing of antibiotics. This less intense, evidence-based } \\
\text { approach achieves the desired objective without wasting resources or } \\
\text { posing risk of harm. }\end{array}$ \\
\hline $\begin{array}{l}\text { De- } \\
\text { intensification }\end{array}$ & $\begin{array}{l}\text { Reducing the intensity of tests and treatments are considered } \\
\text { appropriate options if less invasive or less costly options are sufficient in } \\
\text { achieving specific diagnostic or therapeutic objectives while lessening } \\
\text { the risk of adverse events and reducing burden, costs and } \\
\text { inconvenience for patients. }{ }^{27}\end{array}$ \\
\hline TREATMENT / MANAGEMENT - organisational/team level \\
\hline $\begin{array}{l}\text { Nudge } \\
\text { strategies }\end{array}$ & $\begin{array}{l}\text { Directing (or nudging) clinicians away from overuse by various means } \\
\text { such as publicly declared compacts or default settings in computerised } \\
\text { ordering and prescribing systems, none of which deny clinicians } \\
\text { the choice of taking alternative actions. }\end{array}$ \\
\hline In
\end{tabular}

